
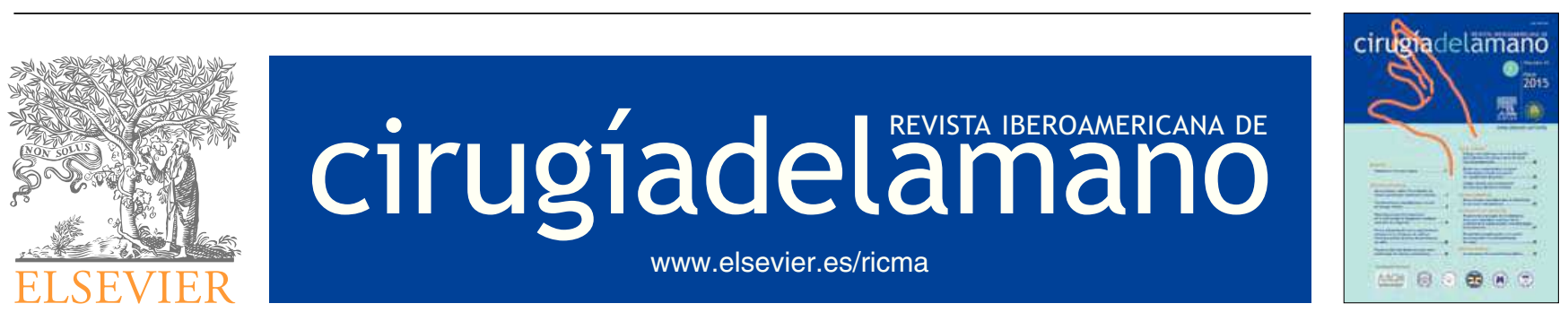

ARTÍCULO ORIGINAL

\title{
Nueva interpretación de los estabilizadores anatómicos en la fractura de muñeca. Parte II: patrones de lesión de las fracturas de radio
}

\author{
J.M. Rotella* y P. Rotella
}

Servicio de Cirugía Ortopédica y Traumatología, Sanatorio del Norte, San Miguel de Tucumán, Argentina

Recibido el 17 de febrero de 2015. Aceptado el 5 de abril de 2015.

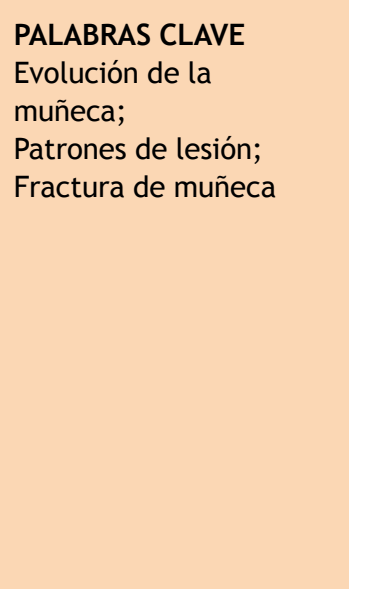

KEYWORDS

Wrist outcomes

Injury patterns

Wrist fracture

\begin{abstract}
Resumen
En este trabajo se presenta una nueva interpretación personal de las lesiones de muñeca basada en el estudio de la filogenia, embriología, anatomía y biomecánica de esta región y en base a esta nueva interpretación planteamos una nueva clasificación (parte I del trabajo), que ayudará a entender los patrones lesionales de las distintas fracturas (parte II del trabajo) y a definir una nueva "estrategia" para el tratamiento de las fracturas de radio (parte III del trabajo).

Se describen tres sectores en la región de la muñeca: cubital (interno); central (medio) y radial (externo); cada uno de ellos presentan diferentes orígenes evolutivo, formativo, constitutivo y funcional.

La semiología radiológica nos permite reconocer que sector de la muñeca está lesionado y cuál es el grado de inestabilidad de cada uno de ellos. Es importante interpretar los diferentes patrones de lesión, punto importante en el diagnóstico de estas lesiones para entender la fisiopatología de las mismas y poder llevar a cabo la correcta estrategia de tratamiento.

๑ 2015 , SECMA. Publicado por Elsevier España, S.L.U. Este es un artículo Open Acces distribuido bajo los términos de la licencia CC BY-NC-ND (http://creativecommons.org/licenses/by-nc-nd/4.0/).
\end{abstract}

New interpretation of anatomical stabilizers in wrist fractures. Part II: Radial fracture injury patterns

\begin{abstract}
A new interpretation of wrist fractures is presented based on the study of the phylogeny, embryology, anatomy and biomechanics of this area. Based on this new interpretation a new classification (Part I) is presented that will help to understand the injury patterns of the different fractures (Part II) and to define a new "strategy" for the treatment of radial fractures (Part III). Three areas in the wrist are described: ulnar (internal); central (middle) and radial (external); each one of them with different evolutionary, formative, constitutional, and functional origins.
\end{abstract}

*Autor para correspondencia.

Correo electrónico: pablorotella@hotmail.com

(c) 2015, SECMA. Publicado por Elsevier España, S.L.U. Este es un artículo Open Acces distribuido bajo los términos de la licencia CC BY-NC-ND (http://creativecommons.org/licenses/by-nc-nd/4.0/). 
The radiography signs allow us to recognize which area is injured, as well as the degree of instability of each. It is important to take into account the different injury patterns, a key point in the diagnosis of these lesions to understand their pathophysiology and thus, to plan a correct treatment strategy.

(c) 2015, SECMA. Published by Elsevier España, S.L.U. This is an open access article under the CC BY-NC-ND license (http://creativecommons.org/licenses/by-nc-nd/4.0/).

\section{Patrones de lesión de las fracturas de radio distal}

Cuando tratamos de interpretar "patrones de lesión" en las fracturas de muñeca, es muy importante ver en las radiografías iniciales, en qué condiciones se encuentra cada uno de los tres sectores de la muñeca (externo o radial, central o medio e interno o cubital) ${ }^{1}$ y el desplazamiento o colapso de los mismos. Esta valoración radiográfica inicial es muy importante en el diagnóstico de la fractura, puesto que nos proporciona información de la magnitud de la energía que provocó el traumatismo y de la dirección de la misma.

En nuestra evaluación de 200 casos clínicos, se detectó que las lesiones, fracturas y desplazamiento de los fragmentos, siguen ciertas reglas y patrones.

La determinación del tipo de trazo de fractura es fundamental en la estrategia de tratamiento para estabilizar cada sector y evaluar cuál es tipo de síntesis a utilizar, dependiendo de la magnitud de la inestabilidad de cada uno.

En este trabajo se va a presentar una interpretación personal de estos patrones de lesión, de las distintas etapas de las fracturas de muñeca y se va a prestar especial atención al sector central.

\section{Sector externo o radial}

Si la conminución de la cortical externa del radio es superior a $3 \mathrm{~mm}$, el desplazamiento coronal en la inclinación radial es mayor y si esta supera los $20^{\circ}$, lleva implícita la lesión del sector interno en un $100 \%$ de los casos (fig. 1).

Si la conminución de la cortical dorsal del radio, compromete los tres tercios de la anchura del radio, conlleva ma- yor inestabilidad y desplazamiento dorsal del radio. Cuando esta conminución supera los $10^{\circ}-15^{\circ}$ de inclinación dorsal, lleva implícita una incongruencia de la articulación radiocubital distal y alteración de los ejes en las articulaciones radiocarpiana y mediocarpiana ${ }^{2}$ (fig. 2 ).

Cuando el trazo de fractura compromete la cortical interna del radio, generalmente el desplazamiento modifica la posición de la carilla sigmoidea del radio y altera la congruencia de la articulación radiocubital distal (fig. 3).

\section{Sector interno o cubital}

El nivel de lesión en la estiloides del cúbito, conlleva diferentes grados de lesión ligamentosa. Cuanto más proximal es la lesión del sector interno, mayor es su inestabilidad, puesto que se compromete y se lesionan mayor cantidad de ligamentos. Cuanto más desplazada está la estiloides

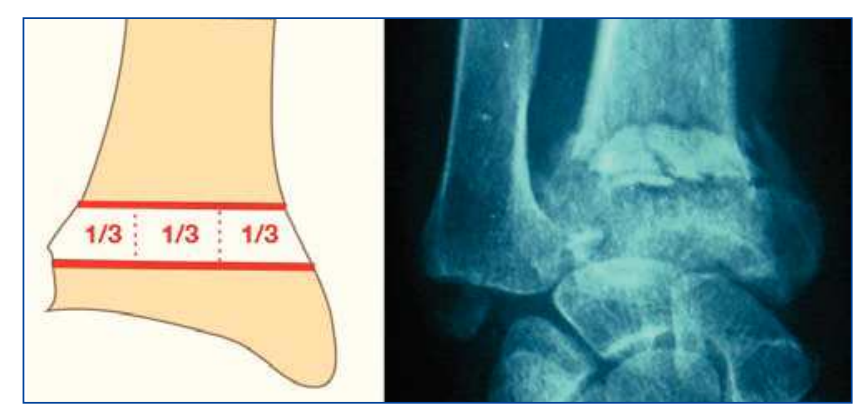

Figura 2 Patrón de lesión del sector radial. Conminución de la cortical dorsal. Se divide el radio en tres tercios.

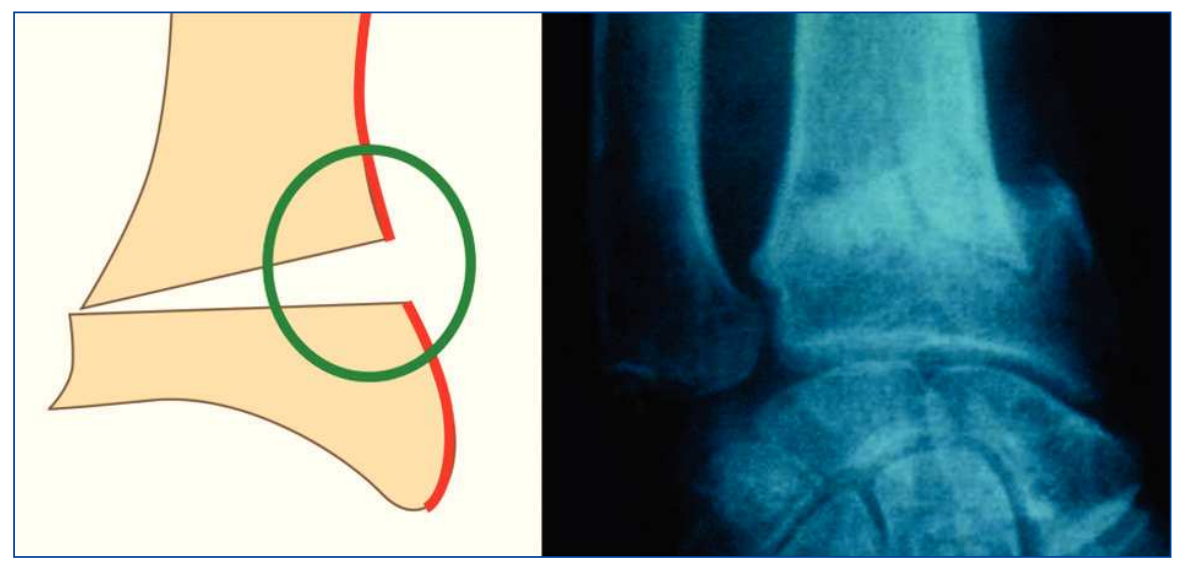

Figura 1 Patrón de lesión del sector radial. Conminución de la cortical externa del radio. 


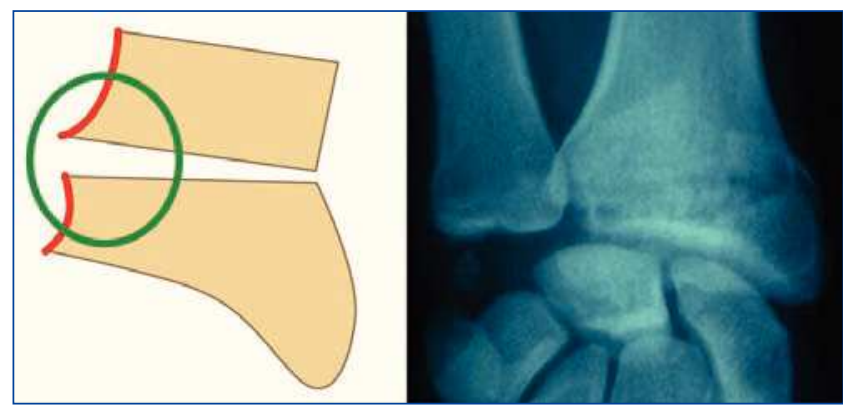

Figura 3 Patrón de lesión del sector radial. Compromiso de la cortical Interna.

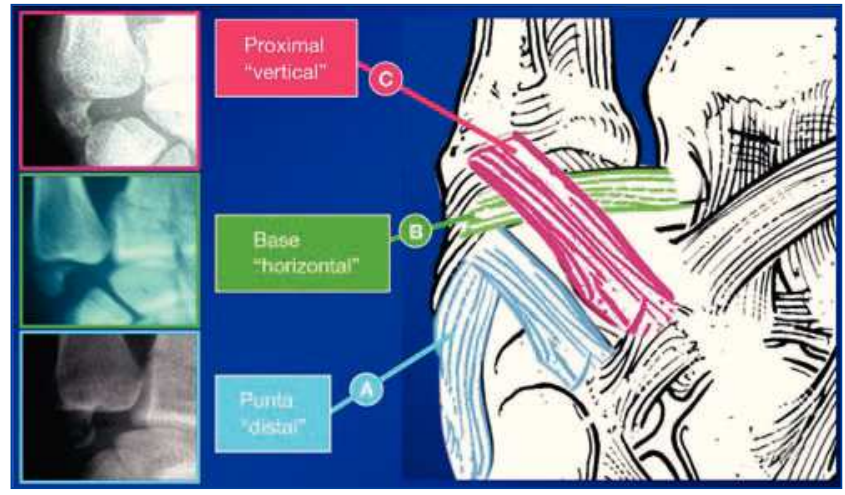

Figura 4 Patrones de lesión del sector interno. Fracturas en la estiloides cubital, existen tres diferentes tipos de patrones.

cubital en la radiografía inicial, mayor es la cantidad de disfunción ligamentosa del sector interno y también se observa una mayor conminución de los otros dos sectores (fig. 4).

Se pueden dividir los distintos patrones de lesión del sector interno en:

a) Fracturas distales o de la punta de la estiloides cubital: en la que se lesionan los ligamentos estilo-carpianos.

b) Fracturas horizontales o de la base de la estiloides cubital: en la que a la lesión anterior, se le agrega la lesión o desinserción del complejo fibrocartílago triangular. c) Fracturas verticales o proximales: en la que a las lesiones anteriores también se le agrega la lesión de los ligamentos cubito-carpianos.

La severidad de la lesión y por lo tanto el grado de inestabilidad del sector interno, va de distal a proximal, cuanto más proximal, más grave es la lesión, más inestable y mayor cantidad de ligamentos alteran su función.

Consideramos que estos diferentes tipos de lesiones, dependen de la posición del carpo en el momento del trauma, como así también de la indemnidad o lesión de los otros dos sectores (central y radial). Generalmente, las lesiones más graves del sector interno, coinciden con los mayores desplazamientos de los otros sectores.

\section{Sector medio o central}

Este sector incluye una parte fija, el tercio interno de la epífisis distal del radio (fosa semilunar) y una parte móvil, la articulación radiocubital distal 1 .

Cuando se evalúa el sector central, puede suceder que su parte fija esté indemne o fracturada. Hemos observado distintos patrones de lesión, dependiendo de que se encuentre fracturado o no.

\section{Sector central sin lesión}

Si la inclinación de la muñeca no fue muy marcada en el traumatismo, se produce una fractura de la estiloides del radio (con o sin fractura del escafoides). Si la inclinación fue mayor, provocará en primer lugar, lesiones ligamentosas intracarpianas y a continuación se afectará el sector interno (fig. 5A).

En el momento del traumatismo, la muñeca está en desviación radial, la primera fila del carpo se traslada hacia cubital y la segunda fila hacia radial, tomando como centro de rotación un punto próximo a la cabeza del hueso grande.

Cuando se produce un traumatismo de muñeca con el sector central sano, la segunda fila, en su traslación hacia radial, producirá la lesión en el sector externo, fracturando la estiloides radia y/o produciendo una fractura de escafoides (fig. 6).

Este mecanismo podría también explicar la frecuente relación que existe entre la fractura de estiloides del radio
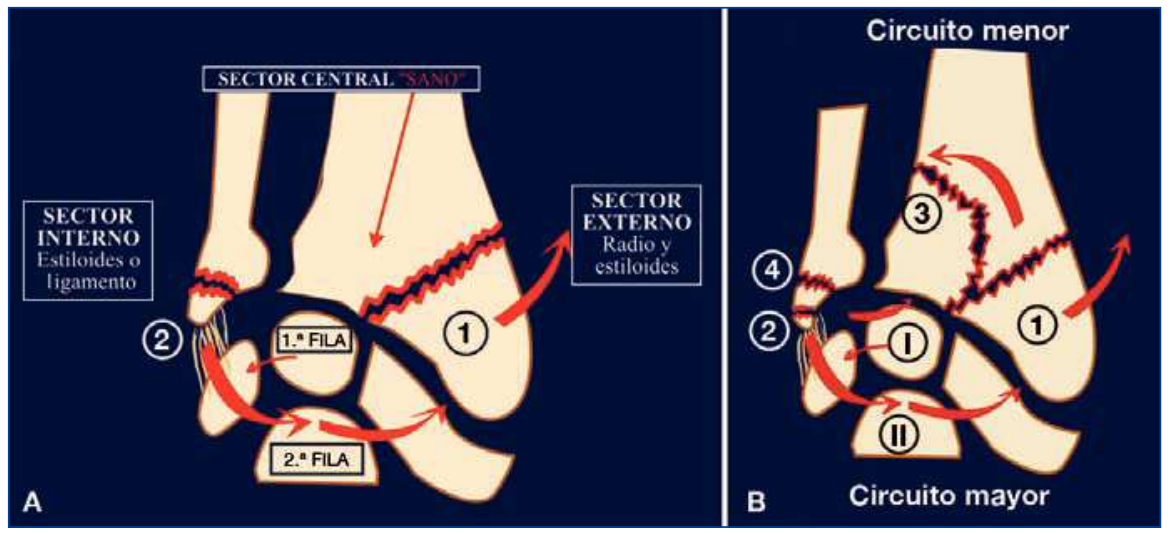

Figura 5 Patrones de lesión del sector central. A. Sector central permanece intacto. B. Sector central lesionado. 


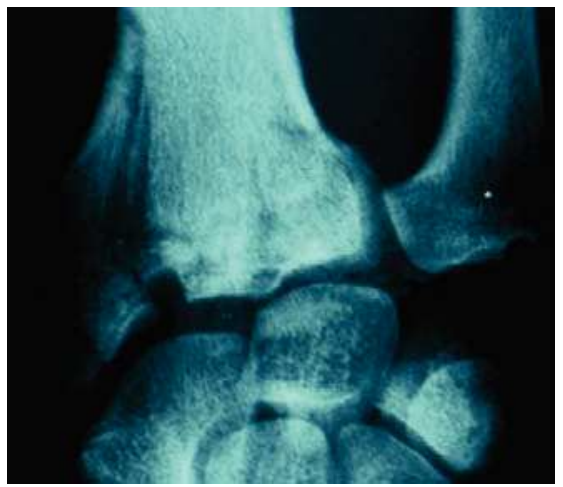

Figura 6 Fractura de muñeca con sector central "sano", en su desplazamiento hacia radial, se produce la lesión en el sector externo, fractura estiloides radial $y / 0$ fractura de escafoides.

con fractura de escafoides y/o la lesión del ligamento escafolunar.

Cuando el sector central, fosa semilunar, está indemne, y el sector radial (estiloides del radio), está muy desplazada, es cuando el sector interno (ligamentos y estiloides del cúbito) presentan las mayores lesiones y desplazamientos. Es porque el sector central (sano) actúa como fulcro de la palanca en la desviación de la muñeca.

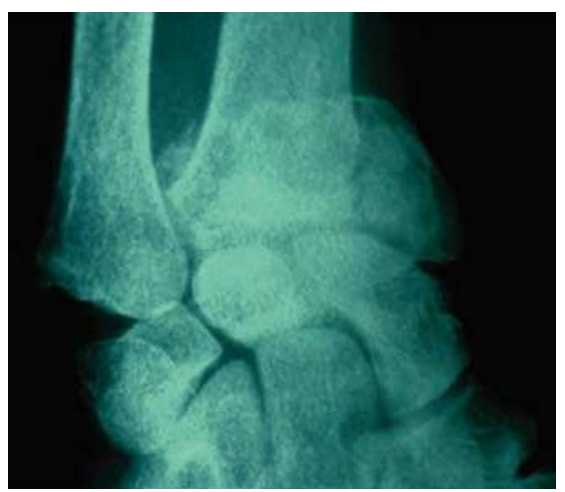

Figura 7 Sector central “lesionado" produce inestabilidad en el "fulcro" que existe a nivel de la articulación radiocubital distal.

\section{Sector central con lesión}

La lesión en el sector central, produce una grave inestabilidad de la muñeca a nivel de la articulación radiocubital distal y se asocia a fracturas conminutas de las superficies articulares, ya sea de la fosa sigmoidea del radio o de la cabeza del cúbito (figs. 5B y 7).

Cuando este sector se lesiona, se lesiona la cortical interna del radio, que permite el ascenso de la fosa del semilunar y produce una alteración en la congruencia de las carillas articulares radiocarpiana y radiocubital distal (fig. 8).

\section{Etapas del traumatismo}

En un traumatismo de muñeca, podemos descubrir dos etapas de lesión.

\section{Primera etapa}

En el impacto de la caída, las energías de las fuerzas son de distal a proximal (fig. 9).

El carpo impacta contra el radio y produce la fractura "intraarticular”. El tipo de fractura dependerá de la posición de la muñeca en supinación o pronación y de la zona de impacto (tenar o hipotenar).

En la mayor parte de las ocasiones, la muñeca y el antebrazo están, en pronación. En esta posición el radio está ascendido y tiene un acortamiento relativo respecto al cúbito. Esto hace que la distribución de fuerzas sea similar en toda la región de la muñeca, tanto en el sector externo como interno.

Si la zona de impacto es en la región hipotenar, la fractura afectará a la fosa semilunar del radio y si es en la región tenar, afectará a la fosa escafoidea del radio. Por tanto, los desplazamientos dependerán del punto de aplicación de las fuerzas (fig. 10).

\section{Segunda etapa}

En esta etapa las fuerzas se dirigen de proximal a distal, ya que el peso del cuerpo comprimirá el radio contra el carpo fijo en el suelo (fig. 11).
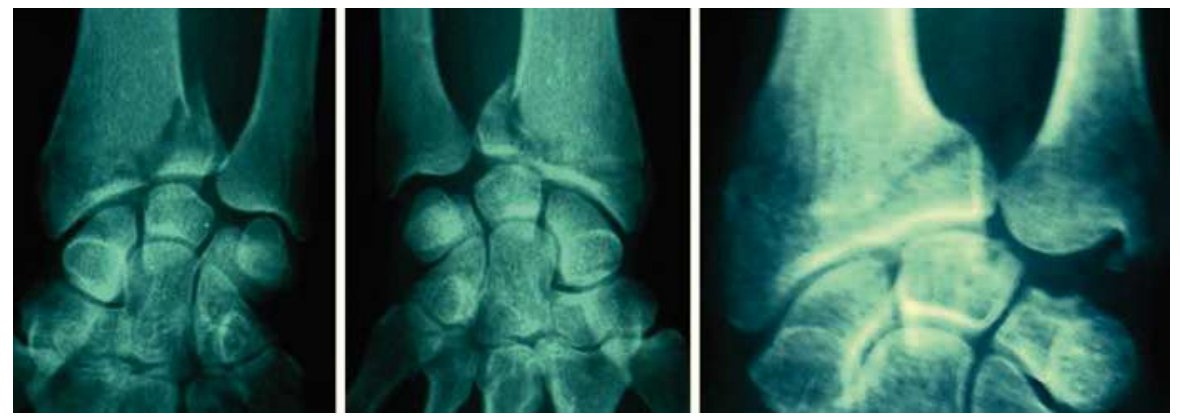

Figura 8 Lesión en la cortical interna, que permite el "ascenso" de la fosa del semilunar y que produce una alteración en la congruencia de las carillas articulares de la articulación radiocubital distal. 


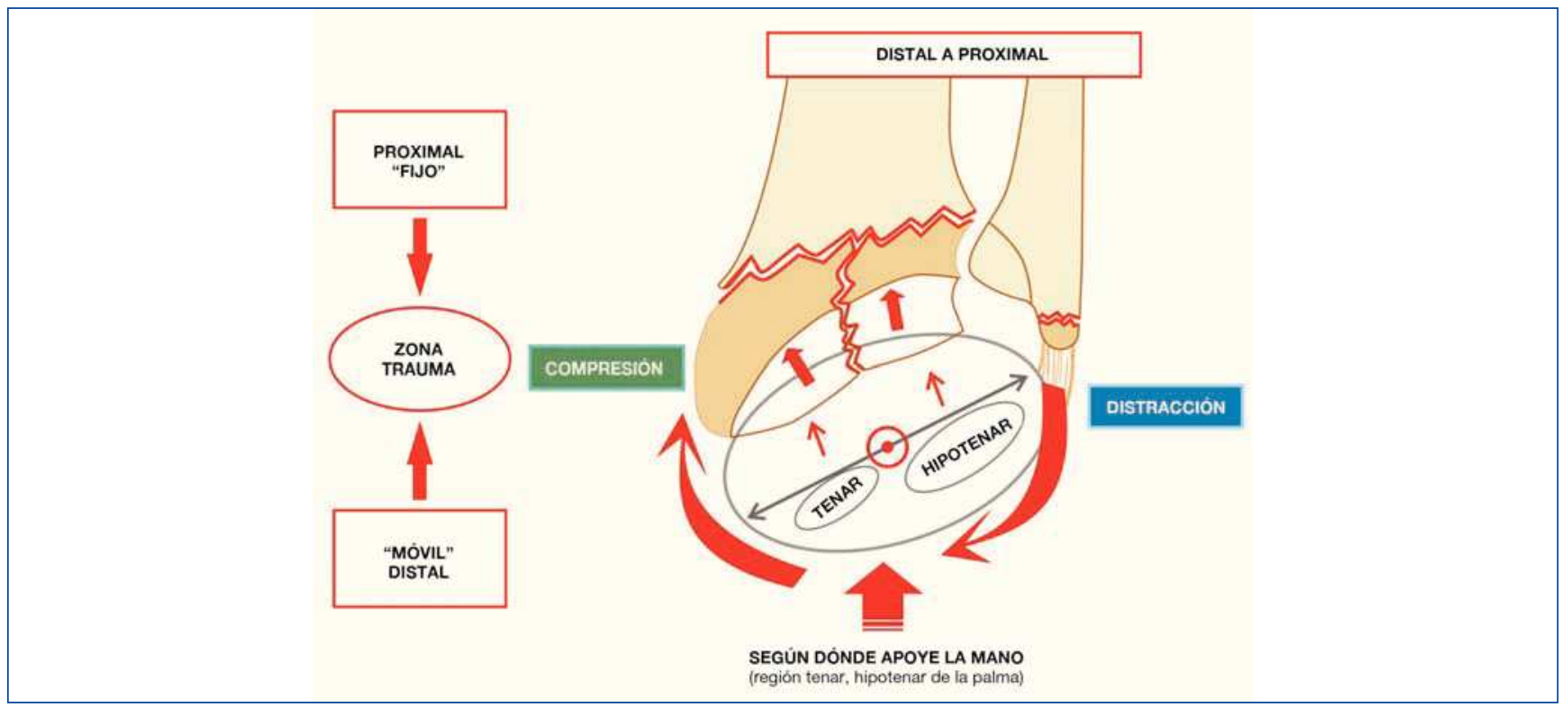

Figura 9 En el momento del trauma, las energías de las fuerzas son de “distal a proximal” y el carpo impacta contra el radio.

En esta segunda etapa se produce la fractura "metafisoepifisaria" extrarticular del radio.

Estas etapas pueden suceder simultáneamente, o de forma aislada. La coexistencia de ambos mecanismos explicaría y sería el responsable de las lesiones intra y extrarticulares.

También tiene mucha importancia y juega un rol fundamental, la calidad ósea del paciente (osteopenia y osteoporosis) en el momento del traumatismo. Esto es un factor determinante, para ver qué zona de la muñeca cede primero en el momento del trauma y así el patrón de fractura.

\section{Importancia y fisiopatología del sector central}

Merece la pena, por su repercusión funcional, prestar especial atención a la lesión del sector central Cuando en la evo- lución surge el sector central, que es la articulación radio-cubital distal ${ }^{1}$, es para que aparezca el movimiento de prono-supinación, fundamental para multitud de actividades.

Muchas fracturas de radio pueden afectar la estabilidad y congruencia de esta articulación. Ambas situaciones, inestabilidad o incongruencia, deben ser tenidas en cuenta ya que pueden comprometer la prono-supinación y ser responsables de dolor cubital de muñeca tras una fractu$\mathrm{ra}^{3-5}$.

Los desplazamientos provocados por una fractura de radio, pueden producir oblicuidades, inclinaciones y acortamientos en la superficie articular de la articulación radiocubital distal que sean causa de incongruencia de esta articulación.

La lesión tanto del sector central como de los otros sectores puede condicionar incongruencia articular y cambios en la orientación de las carillas articulares.

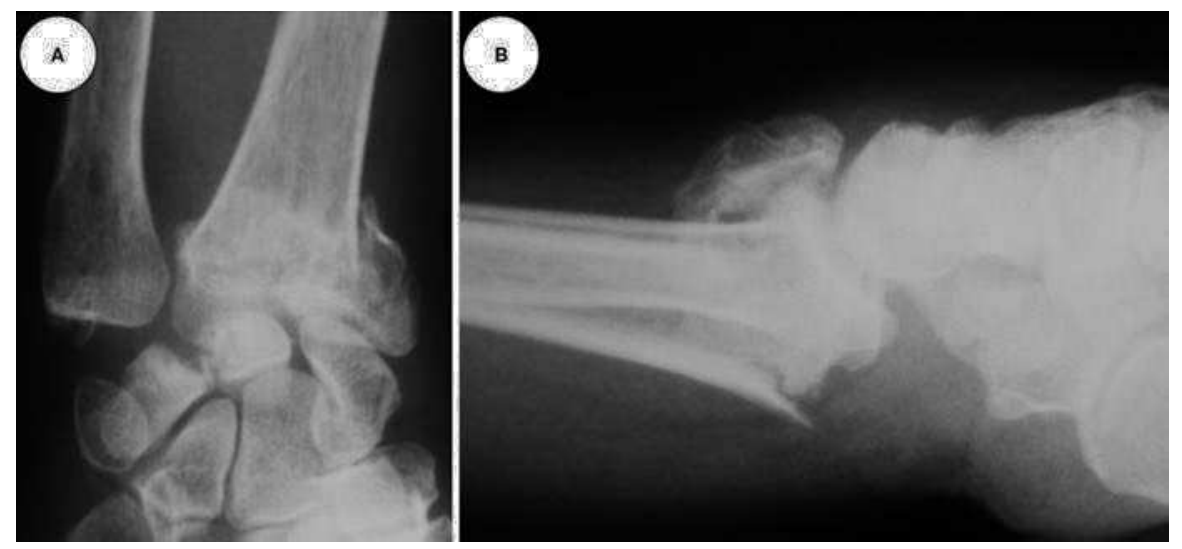

Figura 10 (A) Radiografía antero-posterior, de paciente de 65 años de edad, que presenta fractura intrarticular de muñeca, con acortamiento radial e inclinación externa. El sector central está afectado, con discrepancia de longitud entre el radio y el cúbito por hundimiento de la fosa del semilunar. El sector interno presenta una fractura oblicua proximal de la estiloides del cúbito desplazada; con aumento de la distancia cubito-semilunar y una marcada "translocación" radial del carpo. (B) Radiografía lateral. Se observa una fractura intrarticular, con marcado desplazamiento dorsal de la superficie articular y acortamiento radial por la conminución dorsal. 


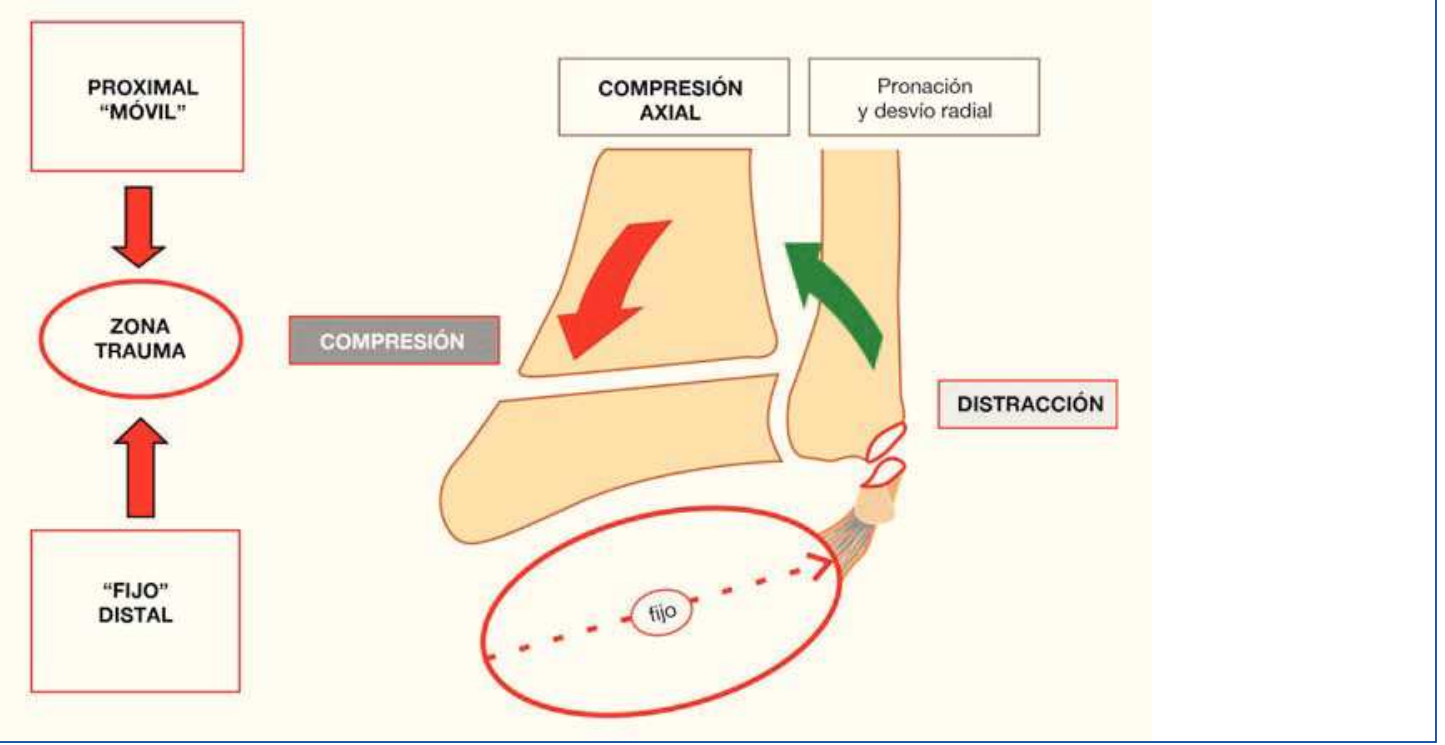

Figura 11 El carpo se encuentra "atrapado e inmóvil" contra el suelo, la continuidad del movimiento del antebrazo sería la responsable de la fractura "metafiso-epifisaria proximal” extrarticular del radio. Energía de proximal a distal.

Pueden existir cuatro zonas de conflictos en el sector central (fig. 12).

Proximal: al perderse el paralelismo de la articulación radiocubital distal, se sobrecarga la zona con el cartílago articular de menor espesor, que es el $1 / 3$ proximal de la cavidad sigmoidea del radio.

Volar: la inclinación dorsal de la epífisis del radio, produce una incongruencia volar de la fosa sigmoidea del radio con el cúbito.

Dorsal: la inclinación volar de la epífisis, produce una incongruencia dorsal de la fosa sigmoidea del radio con el cúbito.
Distal: el acortamiento del radio, produce un cubito plus y un síndrome de impactación cúbito carpiano.

Las distintas zonas de conflicto dependerán del tipo de trazo de fractura en el radio. Se pueden diferenciar cuatro tipos de trazo que tendrán consecuencias distintas en la articulación radiocubital distal.

\section{Inclinación dorsal}

Una fractura transversal del radio, proximal a la articulación radiocubital distal y con una conminución dorsal, con-

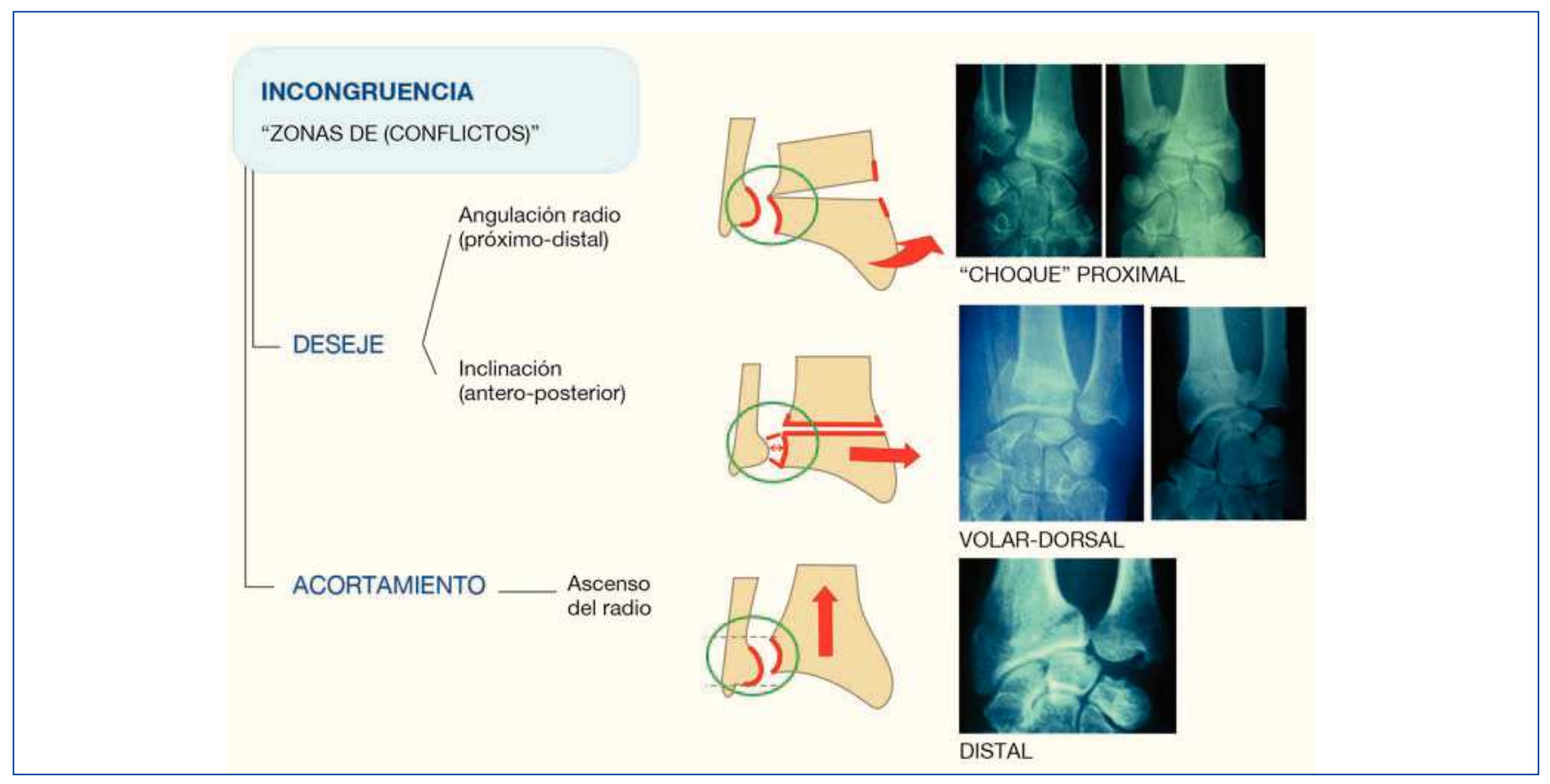

Figura 12 Zonas de conflictos en el sector central. 
diciona una importante inclinación dorsal de la superficie articular del radio.

Este desplazamiento dorsal de la epífisis radial conlleva una incongruencia de la articulación radiocubital distal ${ }^{6-8}$ (sector central). La fosa sigmoidea del radio se inclina hacia dorsal respecto a la cabeza cubital, lo que produce que haya un conflicto de la cabeza del cúbito con el margen volar de la fosa sigmoidea del radio. Este conflicto limitará el movimiento de prono-supinación y producirá dolor cubital en la muñeca.

\section{Transversal}

Una fractura transversal pura, con mínimo desplazamiento volar o dorsal, tiene menos posibilidades de lesión de los sectores interno y medio (fig. 13).

\section{Oblicuo con inclinación radial}

La disminución de la inclinación normal del radio (de $15^{\circ}$ a $22^{\circ}$ ), puede condicionar también una incongruencia de la articulación radiocubital distal. Se produce una inclinación
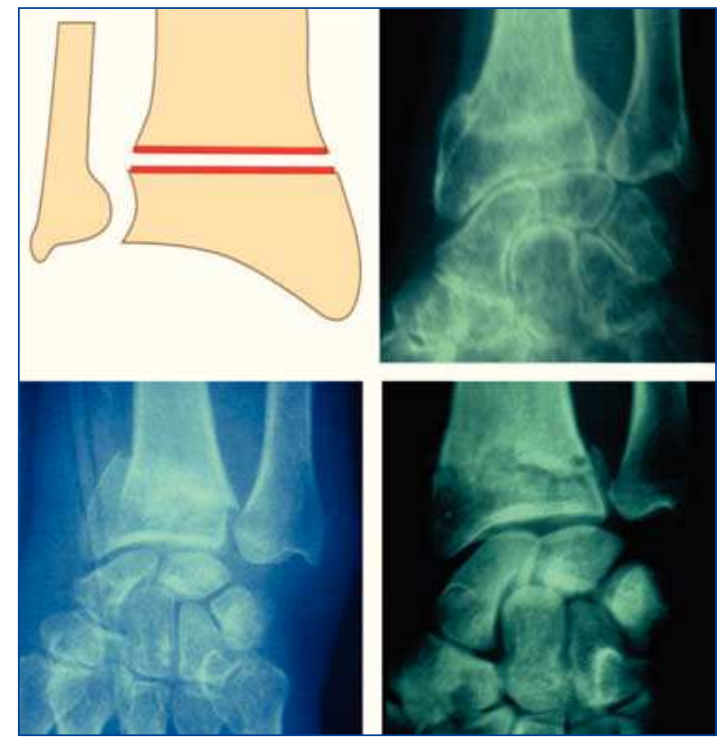

Figura 13 Fracturas transversas que no afectan la articulación radiocubital distal. de la superficie sigmoidea del radio respecto al cúbito, que provoca una diastasas en distal y un conflicto proximal ${ }^{9,10}$ (fig. 14).

La porción de la cavidad sigmoidea que tiene mayor espesor del cartílago, es el margen distal. Con la inclinación de la fosa sigmoidea, se realizan los movimientos de prono-supinación sobre la zona "menos resistente", que es la proximal, que además tiene una concavidad es menor ${ }^{11}$, lo que producirá aún más conflicto (fig. 15).

\section{Fractura que involucra la fosa sigmoidea del radio}

La fosa sigmoidea del radio, se puede dividir en una mitad superior y otra inferior (fig. 16). La mitad inferior es más plana y tiene un mayor espesor su cartílago articular. Si su fractura no condiciona un escalón articular, no crea muchas secuelas.

Si la fractura afecta a la porción superior, que tiene un cartílago de menor espesor y se le añade una angulación dorsal o una inclinación radial de la fosa sigmoidea. Se transfiere el movimiento a la zona articular lesionada, de menor espesor de cartílago y se empeorará el pronóstico y función del sector central.

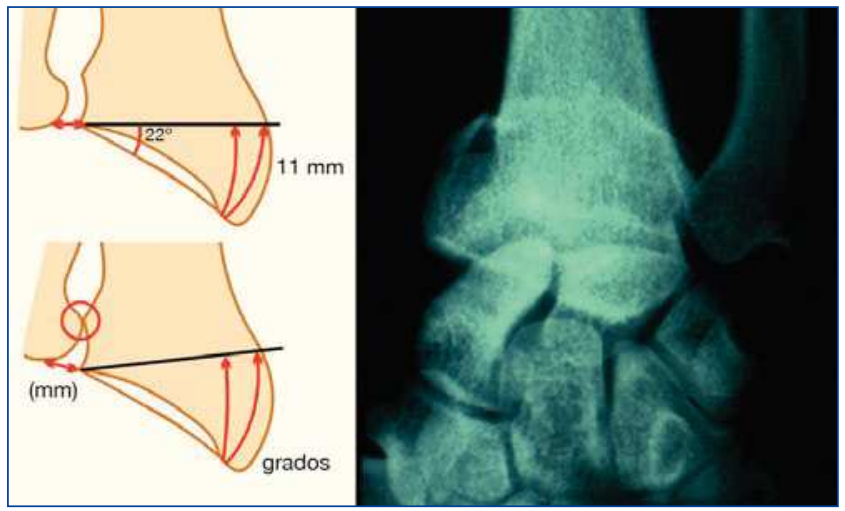

Figura 15 Fractura que afecta a la articulación radiocubital distal, con incongruencia articular. La inclinación y angulación radial provoca un conflicto proximal.

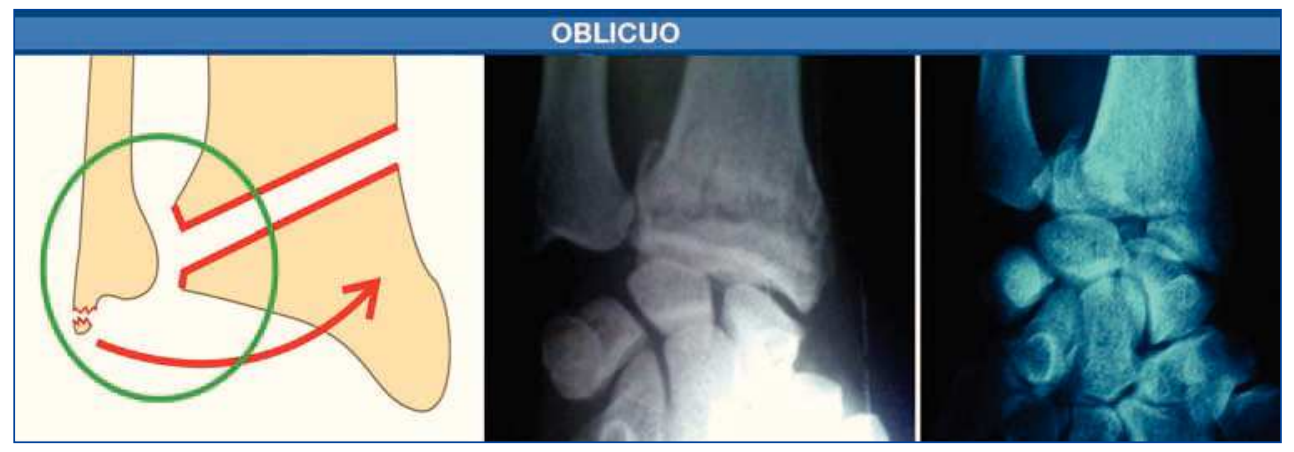

Figura 14 Fracturas oblicuas que afectan la articulación radiocubital distal. Comprometen la superficie articular de la sigmoidea. 


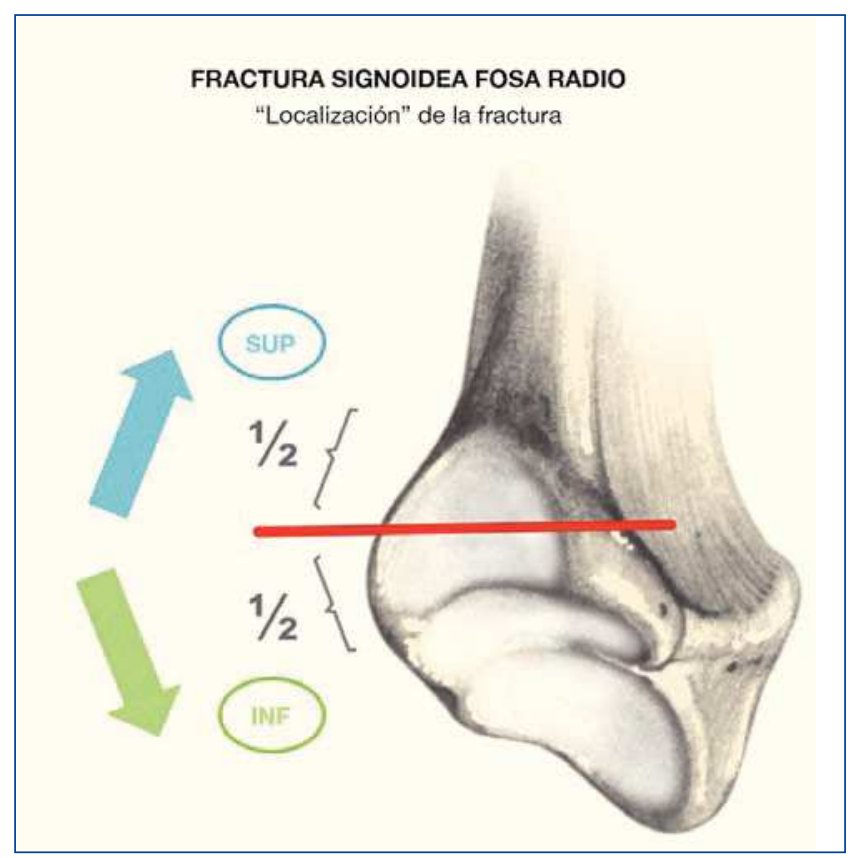

Figura 16 Fractura de la Fosa Sigmoidea el Radio: "Localización de la Fractura" en la mitad superior o inferior.

\section{Conclusión}

Los tres sectores están íntimamente ligados en la biomecánica; una lesión marcada en uno de ellos, conlleva una afectación de los otros sectores. Por eso es muy importante conocer tanto su filogénica y anatomía, como saber interpretar los distintos patrones de lesión que pueden presentar.

\section{Conflicto de intereses}

Los autores declaran no tener ningún conflicto de intereses.

\section{Bibliografía}

1. Rotella JM, Rotella P. Nueva interpretación de los estabilizadores anatómicos en la fractura de muñeca. Parte I: Evolución de la región de la muñeca y nueva clasificación de las fracturas de radio. Rev Iberamer Cir Mano. 2014;42:144-51.

2. Jakob M, Rikli DA, Regazzoni P. Fractures of the distal radius treated by internal fixation and early function. A prospective study of 73 consecutive patients. J Bone Joint Surg Br. 2000;82:340-4.

3. Adams BD. Effects of radial deformity on distal radioulnar joint mechanics. J Hand Surg Am. 1993;18:492-8.

4. Browers WH. Problems of the distal radioulnar Joint. Orthop Surg. 1984;7:289-303.

5. Magart CG. Functional Aspects of the distal radioulnar joint. J Hand Surg [Br]. 1979;4:585.

6. Frykman G. Fracture of the distal radius including sequelaeshouder-hand-finger syndrome, disturbance in the distal radioulnar joint and impairment of nerve function. Acta Orthop Scand. 1967;108:49-153.

7. Park MJ, Cooney WP, Hahn ME, Looi KP, An KN. The effects of dorsally angulated distal radius fractures on carpal kinematics. J Hand Surg Am. 2002;27:223-32.

8. Short WH, Palmer AK, Werner FW, Murphy DJ. A biomechanical study of distal radial fractures. J Hand Surg [Br]. 1987;12:529-34.

9. Aro HT, Koivunen T. Minor axial shortening of the radius affects outcome of Colles' fracture treatment. J Hand Surg Am. 1991;16:392-8.

10. Cooney WP 3rd, Dobyns JH, Linscheid RL. Complications of Colles' fractures. J Bone Joint Surg Am. 1980;62:613-9.

11. Williams PL. Anatomía de Gray. $38 .^{a}$ ed. Madrid: Harcourt-Brace; 1998. 